\title{
Different Strategies for Treating a Colovesical Fistula
}

\author{
Moo Jun Baek \\ Department of Surgery, Soonchunhyang University Cheonan Hospital, Soonchunhyang University College of Medicine, Cheonan, Korea
}

\section{See Article on Page 57-62}

A colovesical fistula (CVF) represents an abnormal communication between the colon and the bladder. Although CVFs are uncommon, they cause significant morbidity and may markedly affect the patient's quality of life. Colovesical fistulae most frequently are the consequence of advanced-stage malignant disease, inflammatory bowel disease, and traumatic or iatrogenic injuries. The diagnosis of a CVF can be challenging and is often delayed for several months after symptoms begin. Colovesical fistulae most commonly occur between the sigmoid colon and the bladder, although cases of fistulae between the small bowel and the ureter have also been reported [1]. Colovesical fistulae are usually more common in men because the uterus acts as a protective barrier in women. Many women with colovesical fistulae have had a previous hysterectomy [2].

The exact method of investigating and treating colovesical fistulae varies considerably from both a geographical and temporal perspective. The authors looked at a series of consecutive patients presenting with colovesical fistulae over a ten year period. They found that despite a similar clinical presentation, colovesical fistulae of various etiologies differed significantly in management and outcome [3]. The author's study is a retrospective one and has a limited numbers of the patients. However, based on the foundation of this study, we can find the right treatment for colovesical fistulae. The study also shows the different methods of treatment that are required, depending on the cause of the colovesical fistula.

\section{CONFLICT OF INTEREST}

No potential conflict of interest relevant to this article was reported.

\section{REFERENCES}

1. Critchely CF. A case of uterto-ileal fistula. Br J Surg 1967;54:812-3.

2. Shatila AH, Ackerman NB. Diagnosis and management of colovesical fistulae. Surg Gynaecol Obstet 1976;143:70-4.

3. Kiani QH, George M, Carapeti E, Schizas A, Williams A. Colovesical fistula: should it be considered a single disease? Ann Coloproctol 2015;31:57-62.
Correspondence to: Moo Jun Baek, M.D.

Department of Surgery, Soonchunhyang University Cheonan Hospital, Soonchunhyang University College of Medicine, 31 Suncheonhyang 6-gil, Dongnam-gu, Cheonan 330-930, Korea

Tel: +82-41-570-3633, Fax: +82-41-571-0129

E-mail: ssurge@sch.ac.kr

(C) 2015 The Korean Society of Coloproctology

This is an open-access article distributed under the terms of the Creative Commons Attribution NonCommercial License (http://creativecommons.org/licenses/by-nc/3.0) which permits unrestricted noncommercial use, distribution, and reproduction in any medium, provided the original work is properly cited. 